\title{
Efficacy and safety of dopamine agonists in patients treated with antipsychotics and presenting a macroprolactinoma
}

\author{
Lucie Allard', Frédérique Albarel(D)2, Jérôme Bertherat ${ }^{3}$, Philippe Jean Caron4, Christine Cortet ${ }^{5}$, \\ Carine Courtillot $^{6}$, Brigitte Delemer ${ }^{7}$, Christel Jublanc ${ }^{8}$, Dominique Maiter ${ }^{9}$, Marie Laure Nunes ${ }^{10}$, \\ Gerald Raverot ${ }^{11}$, Julie Sarfati ${ }^{12}$, Sylvie Salenave', Emmanuelle Corruble ${ }^{13,14}$, Walid Choucha ${ }^{13,14}$ and \\ Philippe Chanson 1 1,15
}

${ }^{1}$ Assistance Publique Hôpitaux de Paris, Service d'Endocrinologie et des Maladies de la Reproduction, Centre de Référence des Maladies Rares de l'Hypophyse HYPO, Hôpital Bicêtre, Le Kremlin-Bicêtre, France, ${ }^{2}$ Assistance Publique Hôpitaux de Marseille, Service Endocrinologie, Centre de Référence des Maladies Rares de l'Hypophyse HYPO, Hôpital de la Conception, Marseille, France, ${ }^{3}$ Assistance Publique Hôpitaux de Paris, Service d'Endocrinologie et Maladies Métaboliques, Hôpitaux universitaires Paris-Centre, Paris, France, ${ }^{4} \mathrm{CHU}$ Toulouse, Endocrinology and Metabolic Diseases, Service d'Endocrinologie, CHU Larrey, Toulouse, France, ${ }^{5} \mathrm{CHRU}$ de Lille, Endocrinology, Lille, France, ${ }^{6}$ Assistance Publique Hôpitaux de Paris, Endocrinology and Reproductive Medicine, Hopitaux Universitaires Pitie Salpétrière-Charles Foix, Paris, France, ${ }^{7} \mathrm{CHU}$ de Reims, Service d'Endocrinologie, Hôpital Robert Debré, Reims, France, ${ }^{8}$ Assistance Publique Hôpitaux de Paris, Endocrinologie, Hôpital de la Pitié-Salpêtrière, Paris, France, ${ }^{9}$ Saint-Luc University Hospital, Endocrinology, Brussels, Belgium, ${ }^{10} \mathrm{CHU}$ de Bordeaux, Department of Endocrinology, Hôpital Haut-Lévêque, 33604 Pessac, France, ${ }^{11}$ Hospices Civils de Lyon, Fédération d'Endocrinologie, Centre de Référence des Maladies Rares de I'Hypophyse HYPO, Groupement Hospitalier Est, Bron, France, ${ }^{12}$ Assistance Publique Hôpitaux de Paris, Endocrinology, Hôpital Saint-Antoine, Paris, France, ${ }^{13}$ Assistance Publique Hôpitaux de Paris, Service HospitaloUniversitaire de Psychiatrie et Addictologie, Hôpitaux Universitaires Paris-Saclay, Hôpital Bicêtre, Le Kremlin-Bicêtre, France, ${ }^{14}$ Université Paris-Saclay, Univ. Paris-Sud, CESP, Equipe MOODS, Le Kremlin-Bicêtre, France, and ${ }^{15}$ Université Paris-Saclay, Univ. Paris-Sud, Inserm, Signalisation Hormonale, Physiopathologie Endocrinienne et Métabolique, Le

\begin{abstract}
Context: In patients treated with antipsychotics, the rare occurrence of a macroprolactinoma represents a therapeutic challenge.

Objective: Our aim was to evaluate the efficacy and psychiatric safety of dopamine agonists (DAs) prescribed for large macroprolactinomas in patients with psychosis treated with antipsychotics.
\end{abstract}

Design: This was a multicenter (France and Belgium) retrospective study.

Patients: Eighteen patients treated with antipsychotics were included.

Results: Under DA, median PRL levels decreased from 1247 (117-81 132) to 42 (4-573) ng/mL ( $P=0.008)$, from 3850 (449-38 000) to 141 (60-6000) ng/mL ( $P=0.037)$ and from 1664 (94-9400) to 1215 (48-5640) ng/mL $(P=0.56)$ when given alone $(n=8)$, before surgery $(n=7)$, or after surgery $(n=6)$, respectively. The prolactinoma median largest diameter decreased by $28 \%(0-57)$ in patients under DAs alone $(P=0.02)$ but did not change when given after surgery. Optic chiasm decompression was achieved in $82 \%$ of patients. Five patients $(28 \%)$ were admitted for psychotic relapse while receiving DAs (but three of them had stopped antipsychotic treatment at that time). A more severe underlying psychosis, rather than the DA treatment itself, may explain such psychiatric admissions.

Conclusion: Even if the DA efficacy on PRL levels and tumor volume in patients with macroprolactinoma under antipsychotic drugs is less impressive than that typically observed, it may be considered satisfactory for half of our patients, particularly in cases of optic chiasm compression. Psychotic exacerbation was unusual in these patients, occurring mostly in those with the most severe psychotic forms. DAs may therefore be used as antitumor treatment for macroprolactinoma in patients with visual involvement, severe headaches or invasion into the skull base who receive antipsychotics.

European Journal of Endocrinology

(2020) 183, 221-231
Published by Bioscientifica Ltd. 


\section{Introduction}

While microprolactinomas are quite prevalent in the general population, macroprolactinomas are much rarer (1). Due to their size, these macroadenomas may be responsible for mass effects, particularly optic chiasm compression. Fortunately, in these cases, dopamine agonists (DAs) are able not only to decrease prolactin (PRL) levels but also to allow tumor shrinkage, alleviating compression symptoms. DAs act by binding to the dopamine type 2 (D2) receptor at the level of the lactotroph cell.

Psychotic disorders are also rare since their incidence is 26.6 per 100000 person-years (2) and their prevalence is approximately 4 per 1000 (3). Their treatment is based on antipsychotic drugs that act by blocking or mitigating the activity of dopamine at the level of the dopamine type 2 receptor (D2R) in the mesolimbic pathway as well as the nigrostriatum and tubuloinfundibular system $(4,5)$. Treatment with antipsychotics, particularly older typical or first-generation drugs, has a propensity to induce hyperprolactinemia caused by the blockade of D2R at the level of pituitary lactotroph cells (tubuloinfundibular pathway) (6). The second-generation or atypical antipsychotics, which dissociate more quickly from the D2R (7), induce less hyperprolactinemia $(8,9)$.

In patients who require both antipsychotics for mental disorders and DAs for hyperprolactinemia, one can legitimately question whether, due to their opposite effects on the D2R, DAs could make antipsychotics ineffective and lead to psychotic exacerbation, while antipsychotics could make DAs less effective on PRL levels but also on prolactinoma mass. Therefore, in patients with severe psychotic conditions treated with antipsychotics, the rare occurrence of a macroprolactinoma, particularly when it compresses the optic chiasm, represents a therapeutic challenge.

Few case reports have suggested a risk of emergence or worsening of psychosis when patients under antipsychotics are treated with DAs (10, 11, 12, 13). However, there are no prospective or retrospective studies evaluating the efficacy of DAs on macroprolactinomas.

This led us to evaluate in a retrospective study how these patients were managed in French and Belgian university hospitals to evaluate the efficacy of DAs on the PRL concentration, adenoma volume and visual impairment and to assess the psychiatric risk linked to DA prescription in these patients treated by antipsychotics for their mental disorders.

\section{Methods}

We contacted various endocrinology centers in university hospitals in France and Belgium (thanks to the Club Français de l'Hypophyse, a national working group of the French Endocrine Society) and collected data about the medical background, endocrine pathology, treatments, psychiatric disease course, including worsening episodes and admission, from the charts of each individual patient with psychiatric disease and a macroprolactinoma known to have received treatment with antipsychotics and DAs. We focused on the period between 1987 (availability of MRI in France) and 2017.

We specifically collected the initial characteristics of the prolactinoma: size on MRI, PRL level, and results of visual assessment. Then, we examined the sequence of the treatment of prolactinoma: first-line treatment, surgery or DAs; second-line treatment, DAs or surgery, and eventually radiotherapy. At each step, we analyzed the efficacy of DAs on serum PRL levels, prolactinoma size and visual impairment. The same criteria were applied for the efficacy of surgery and radiotherapy. We also considered the effect of treatments on chiasm compression and cavernous sinus invasion when specified. Finally, we examined the doses of DAs at the time of best control of PRL levels and prolactinoma size to compare them with those used in the population of 'usual' macroprolactinomas treated with DAs but not with antipsychotics.

As for the psychiatric tolerance of DAs, we collected all intercurrent events that could be associated with a worsening of the mental disorder: hospital admissions, accepted by the patient or compulsory, self-harming, aggressiveness, and urgent antipsychotic change. Then, we tried to evaluate the link between DA use and psychotic exacerbation by investigating previous hospital admissions, psychosis severity, the number of treatments before the introduction of DAs and whether DAs were truly taken by the patient at the precise moment of psychiatric impairment.

Finally, we sought to determine whether endocrine treatment decisions were influenced by psychiatric risk or only by the evolution of macroprolactinoma.

\section{Statistical analysis}

Data are expressed as medians (ranges). Differences between two subgroups were analyzed using the Wilcoxon test or Mann-Whitney $U$-test (in the case of 
a nonparametric distribution). Nominal variables were analyzed using Fisher's exact test.

The study was not approved by a medical ethical or institutional review board, as it was not deemed necessary by French law for observational non-interventional studies on humans, which indicates that clinical and biological data obtained during usual medical care can be used for future research. When obtaining these data, each patient is warned that he has the opportunity to refuse the use of his/her data for future research. For University hospitals involved in this study, the 'Commission Nationale Informatique et Liberté' gave the agreement for the use of medical data in this particular context of retrospective studies and non-opposition of patients.

\section{Results}

\section{Characteristics of the patients}

We collected the endocrine clinical charts of 18 (12 M, 6F) patients with a mean ( \pm s.D.) age of $45 \pm 14$ years from several hospitals in France and Belgium (Le Kremlin-Bicêtre, Paris, Marseille, Lyon, Bordeaux, Toulouse, Reims, Lille, Brussels). Macroprolactinomas were diagnosed between 1987 and 2017, and the mean follow-up was $9 \pm 10$ years (Table 1 ). Fifteen patients were still actively followed, one was deceased in 2009, and two were lost to follow-up in 2013 and 2015. Psychiatric disorders included schizophrenia in five cases, chronic psychosis in three, bipolar disorder in two, and autism in one case, while the diagnosis was imprecise (behavior disorder, delusional and depressive syndrome, anxiety disorder) in five others. Sixteen patients with a known high risk of hyperprolactinemia induction were taking antipsychotics and six had taken, alone or in association, antipsychotics that are not known to be associated with hyperprolactinemia. Symptoms leading to discover the prolactinoma were headaches in four patients, visual problems in four patients, amenorrhea in four patients, galactorrhea in three patients, sexual problems in two patients, seizures in one patient and cognitive impairment in one patient. The discovery was fortuitous in three patients. All the patients except one had hypogonadism.

\section{Efficacy of DAs in patients with macroprolactinoma also treated with antipsychotics.}

Efficacy on the PRL levels

Every patient received DAs, either alone or before or after surgery or radiotherapy. The therapeutic sequences are described in Fig. 1. We divided the patients into two groups: those who were only treated with DAs $(n=8)$ and those who were also treated with surgery or radiotherapy $(n=10)$. In this latter group, the effect of DAs was studied, when given, both before surgery and after surgery.

As expected, the best response to DAs in terms of PRL levels (Fig. 2) was found in the patients who were only treated with DAs: PRL levels normalized (normal PRL levels are less than $25 \mathrm{ng} / \mathrm{mL}$ ) in two patients (25\%), decreased by $>50 \%$ in seven patients $(87.5 \%)$, and by $<50 \%$ in one patient $(12.5 \%)$. All these patients were treated with antipsychotics at the time of DA treatment. The average decrease in PRL concentration was 94\%. The initial median PRL level was 1247 ng/mL (range, 117-81 132), whereas the minimal median PRL level after DA treatment was $42 \mathrm{ng} / \mathrm{mL}(4-573)(P=0.008)$. The average cabergoline dose needed to achieve the best biochemical control was $0.75 \mathrm{mg}$ per week.

In the group treated with DAs before surgery or radiotherapy $(n=7)$, PRL levels also decreased by $>50 \%$ in five patients and by $<50 \%$ in one patient (Fig. 2). Data are missing for one patient (Patient \#11). The average PRL level decrease was $85 \%$. The initial median PRL level was $3850 \mathrm{ng} / \mathrm{mL}$ (449-38 000), whereas the minimal median PRL level under DA treatment was $141 \mathrm{ng} / \mathrm{mL}$ (60-6000) $(P=0.03)$. The average cabergoline dose needed to obtain the best biochemical control was $1.375 \mathrm{mg}$ per week.

In the group treated with DAs after surgery or radiotherapy $(n=6)$, PRL levels decreased by $<50 \%$ in four patients and increased in two patients (Fig. 2). The initial median PRL level was 1664 ng/mL (94-9400), whereas the minimal median PRL level after DA treatment was $1215 \mathrm{ng} / \mathrm{mL}$ (48-5640). The average cabergoline dose needed to obtain the best biochemical control was 1.65 mg per week.

The initial and minimal PRL levels achieved in each individual, according to his/her subgroup, and the corresponding DA dose for achieving this result are illustrated in Fig. 2. The patients who had the best biochemical response to DAs were those who did not require surgery or radiotherapy. When one of these interventions was necessary, the response was better before than after the procedure.

Secondary analysis restricted to the 16 patients treated with neuroleptics known to increase PRL levels gave similar results (see Supplemental data, see section on supplementary materials given at the end of this article).

A change for antipsychotics causing less effects on D2R was attempted in six patients (replacement of olanzapine by clozapine and thereafter by aripiprazole in Patient \#1, 

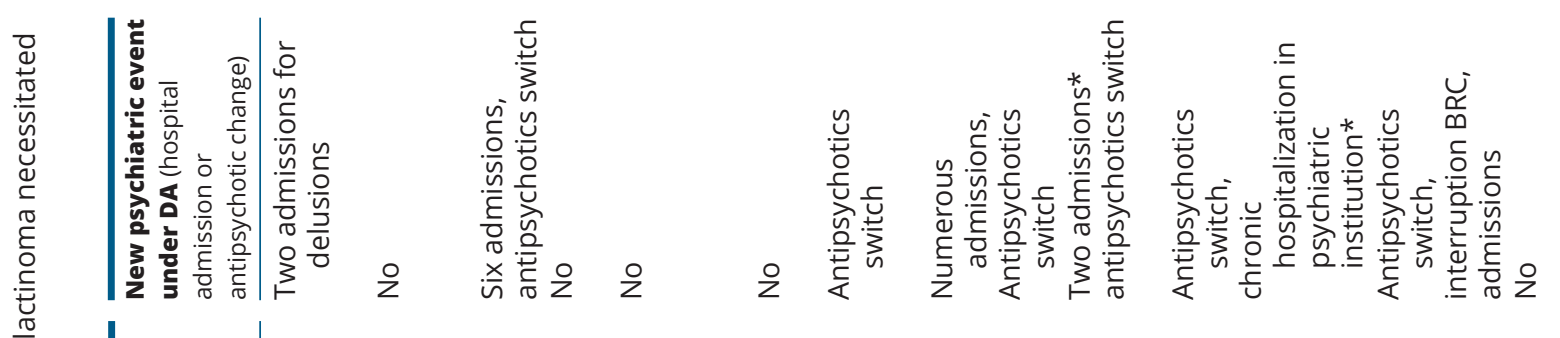

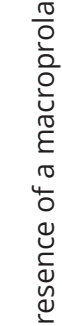

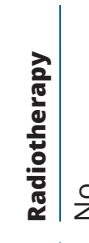

$\stackrel{2}{2} \stackrel{2}{2} \frac{\circ}{2}$

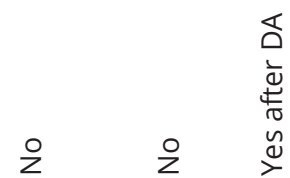

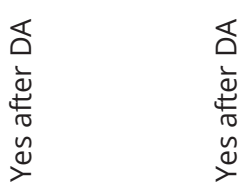

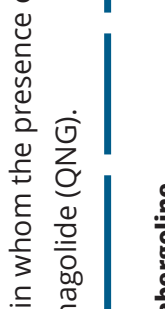

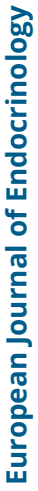

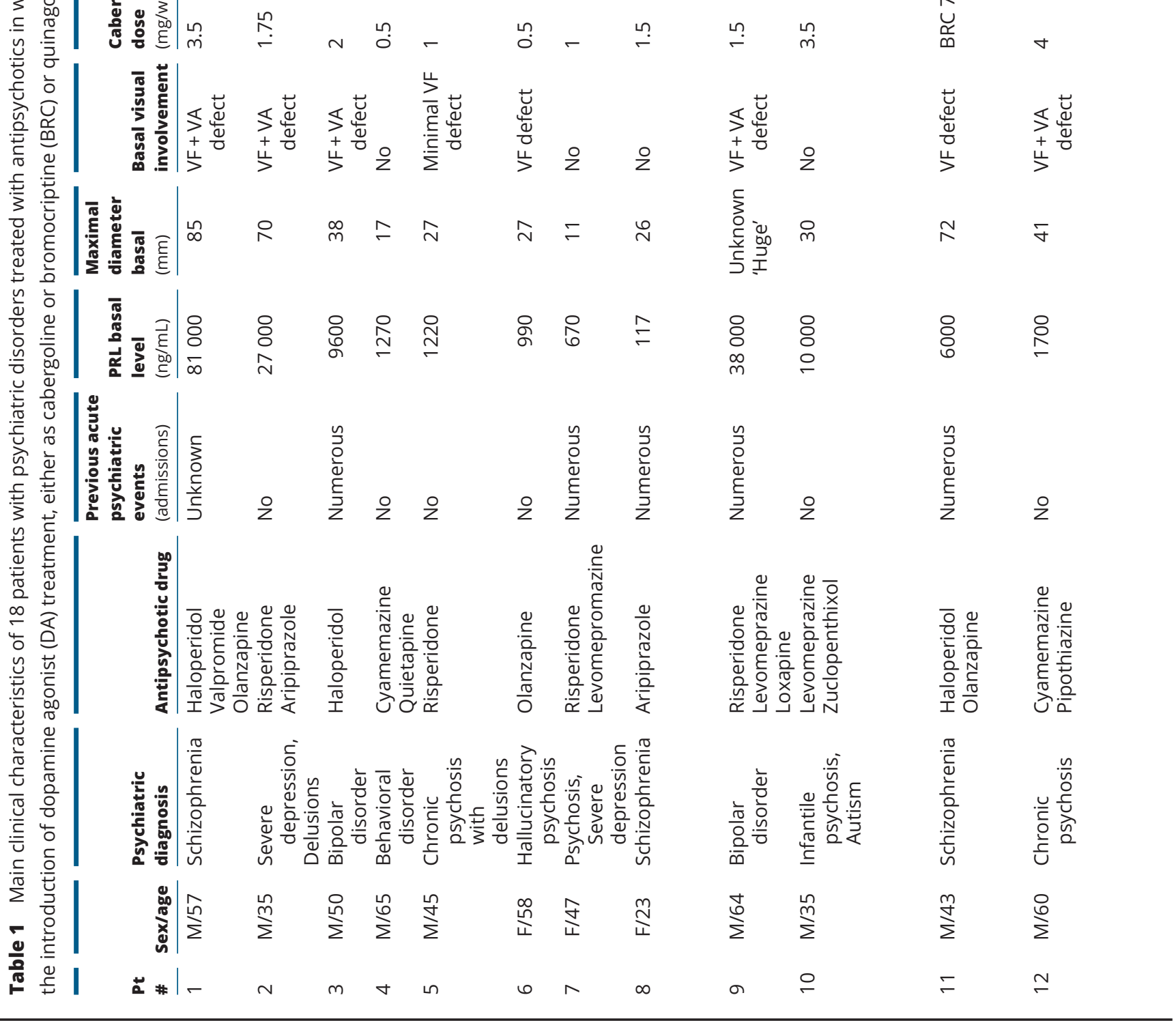




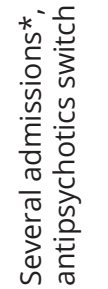

$\frac{n}{\frac{n}{0}}$
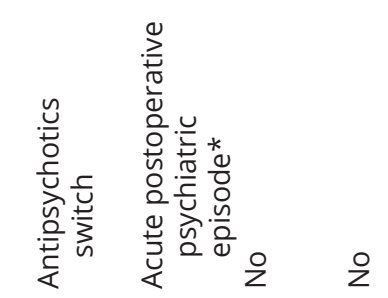

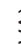

齐号

兽

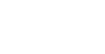

$\circ \stackrel{m}{\square} \infty \stackrel{\square}{\circ}$

$-\stackrel{m}{-}$$$
\text { (n) }
$$

of haloperidol by clozapine in Patient \#3, of olanzapine for clozapine in Patient \#6, of risperidone for aripiprazole in Patient \#7, of risperidone for quietapine in Patient \#9, and of risperidone by quietapine in Patient \#14) which did not alter the efficacy of of DAs on PRL levels.

\section{Efficacy on tumor size}

The response of tumor size (the largest diameter) to DAs tended to be better in nonoperated patients (Fig. 3).

In the group of patients only treated with DAs $(n=8)$, the initial median of the largest diameter of the adenoma was $27 \mathrm{~mm}$ (11-87), which decreased to $24 \mathrm{~mm}$ (11-37) under DAs $(P=0.02)$. Two patients had a $>50 \%$ decrease in the tumor diameter, two had a $25-50 \%$ decrease, three had a $5-25 \%$ decrease, and one did not change his tumor volume (Fig. 3). The larger prolactinomas tended to respond better to DAs than the smaller ones. Abutment to optic pathways that were visible on MRI in four patients before DA disappeared after DA treatment in three of them.

In the group treated with DAs before surgery or radiotherapy $(n=7)$, the initial median of the largest diameter of the adenoma was $22 \mathrm{~mm}(10-72)$. The macroprolactinoma shrunk impressively ( $80 \%$ decrease in maximal diameter) in one patient (Patient \#11), whereas it remained unchanged in four others, and data were not available for Patient \#9 (Fig. 3). Four patients had a chiasmal compression visible on MRI before DA treatment, which persisted in three of them under DA treatment.

In the patients treated with DAs after surgery $(n=6)$, the median largest adenoma diameter was $19 \mathrm{~mm}(5-42)$ initially and $12 \mathrm{~mm}(5-43)$ after DAs, but none of the prolactinomas showed a significant decrease under DAs after surgery (Fig. 3), and no change in terms of chiasmal compression was observed on MRI.

Secondary analysis restricted to the 16 patients treated with neuroleptics known to increase PRL levels gave similar results (see Supplemental data).

There was no correlation between PRL levels decrease and tumor shrinkage $(r=0.21)$.

\section{Efficacy on visual defects}

The course of the visual fields is illustrated in Fig. 4. In the group of patients only treated with DAs, visual field defects disappeared in three patients under DAs: quadranopia in two cases (Patients \#2 and \#7) and blind spot exclusion in the third case (Patient \#6). The only patient who had hemianopia kept it despite DA treatment (Patient \#1), but 


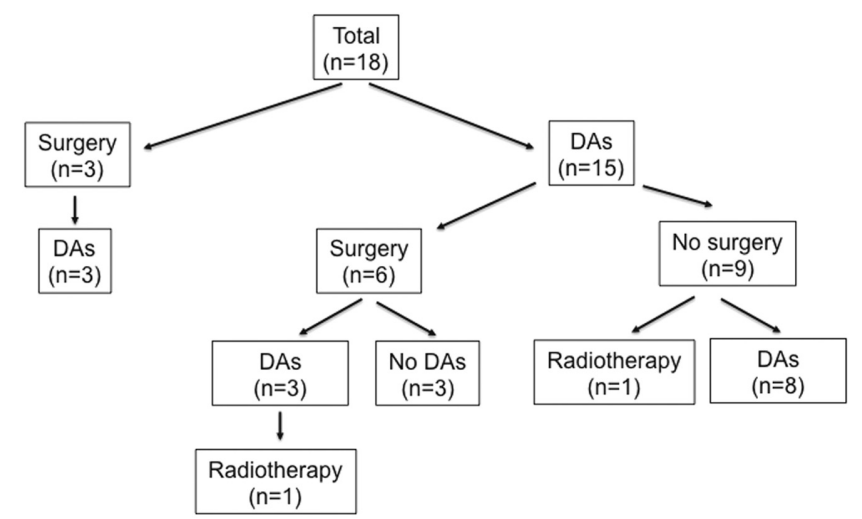

Figure 1

Sequences of treatment in the 18 patients with psychotic disorders treated with antipsychotics and a macroprolactinoma necessitating DA

the relation to adenoma compression remained uncertain. Visual acuity globally improved in patients only treated with DA.

Missing data impaired the precise interpretation of the visual parameters in patients treated with DAs before or after surgery or radiotherapy. Visual fields and acuity did not seem to improve significantly.

\section{Efficacy of surgery in patients with macroprolactinoma also treated with antipsychotics}

Surgery was performed as first-line treatment in three patients for fear of decompensating the underlying psychotic disorder by using DAs: these are generally oldest records. Surgery was performed as second-line treatment after a trial of DA in six cases because the effect of DA was considered as not optimal or due to loss of compliance.

The median PRL level was $1137 \mathrm{ng} / \mathrm{mL}$ before surgery and decreased to $115 \mathrm{ng} / \mathrm{mL}$ (lowest PRL levels) in the short term after surgery (median at 1.8 months postoperatively). Nevertheless, over time, PRL levels increased again in almost every patient, reaching a median level of $583 \mathrm{ng} /$ $\mathrm{mL}$ at a median duration of 6 years after surgery. Surgical treatment reduced tumor size substantially in the seven patients with available data: the median reduction in maximal diameter was 50\%.

Among the nine surgical patients, six (66\%) needed complementary treatment with DAs after surgery.

Time frame of PRL levels and tumor reduction as well as DA doses and type of antipsychotic drugs are given in detail for each patient on an individual figure provided in Supplemental data.

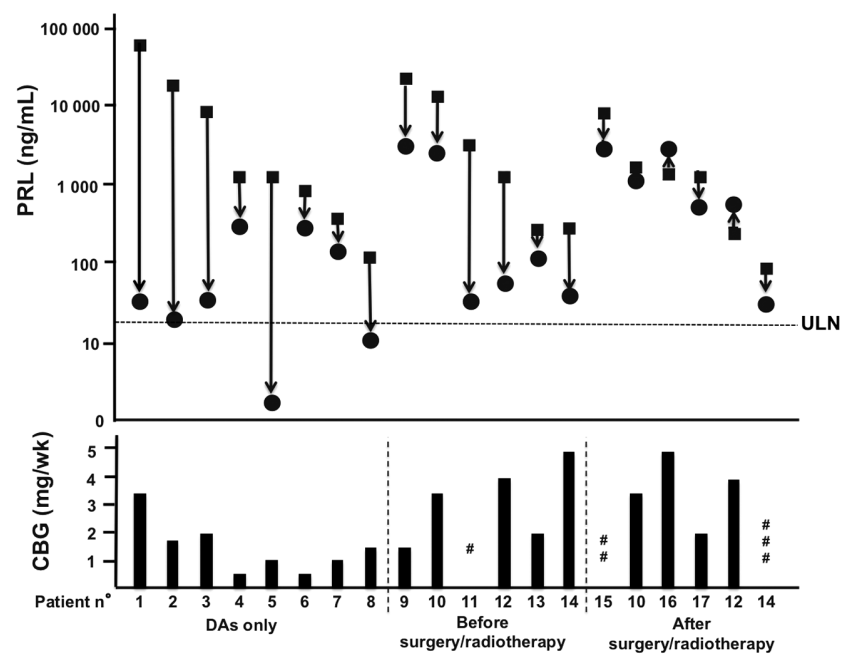

Figure 2

Individual course of PRL levels under dopamine agonists (DAs) in three groups of patients: those who were treated by DAs only, those treated with DAs before surgery or radiotherapy and those treated by DAs after surgery or radiotherapy. The initial PRL level is indicated by a closed square, and the lowest PRL level achieved under DA is indicated by a closed circle. For each individual, the dose of cabergoline (CBG) necessary to achieve the best result in terms of tumor size is given in the lower pannel. Three patients received bromocriptine at a daily dose of $7.5 \mathrm{mg}$ (\#) or $15 \mathrm{mg}$ (\# \# \#) or quinagolide at a daily dose of $225 \mu \mathrm{g}$ (\# \#). Note that the scale of PRL levels is logarithmic. ULN, upper limit of normal of PRL levels (25 ng/mL).

\section{Psychiatric tolerance of DAs}

Throughout the history of the 18 patients, nine presented psychosis relapses requiring admission in psychiatric units (Table 1). Four of these nine patients did not take DAs at the time of psychiatric relapse, which was concomitant of a surgical operation (Patients \#9, \#13 and \#16) or occurred 10 years after cabergoline interruption (Patient \#10). Among the five remaining patients taking DAs at the time of psychiatric relapse (Patients \#1, \#3, \#8, \#11, and \#14) (28\% of all patients), three did not take any antipsychotic at the time of psychiatric crisis (Patients \#8, \#11, and \#14). When these two groups (the nine patients admitted to the psychiatric unit and the nine patients not admitted) were compared, we noticed that the patients who needed admission had a more severe psychiatric disease. Indeed, among them, eight (89\%) had a previous psychiatric admission vs only one (11\%) in the group of nine patients not admitted $(P=0.0034)$. The overall number of drugs used for psychiatric treatments was also higher in the group of patients requiring admission to a 


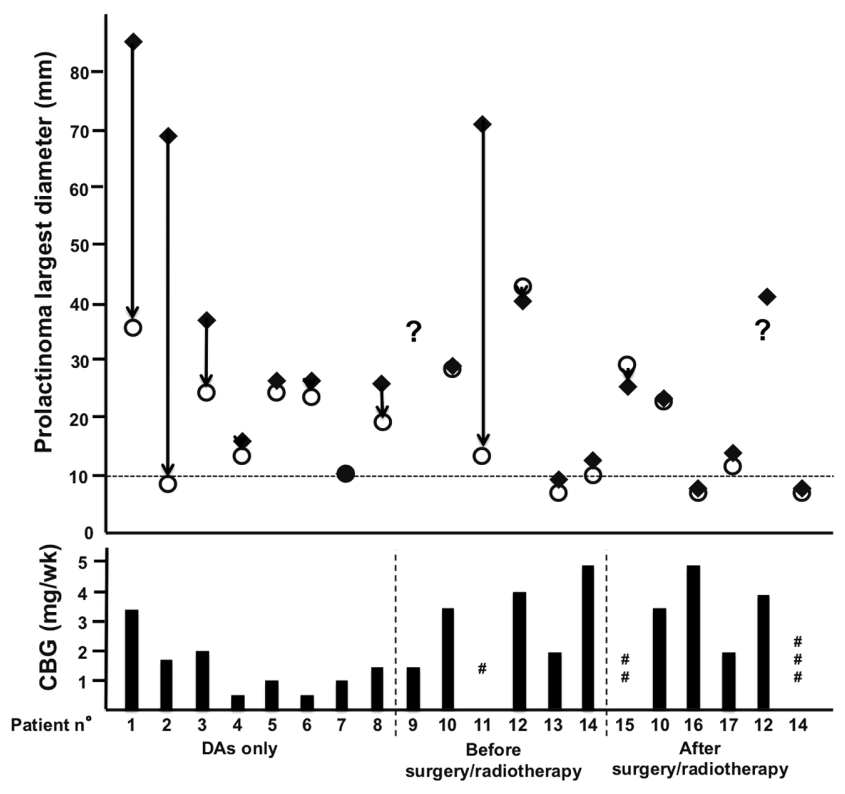

Figure 3

Individual course of the size of the macroprolactinoma (given as the largest diameter) in three groups of patients: those who were treated by dopamine agonists (DAs) only, those treated with DAs before surgery or radiotherapy and those treated by DAs after surgery or radiotherapy. The initial maximal diameter is indicated by a closed diamond, and the lowest maximal diameter achieved under DA is indicated by an open circle. For each individual, the dose of cabergoline (CBG) necessary to achieve the best result in terms of tumor size is given in the lower pannel. Three patients received bromocriptine at a daily dose of $7.5 \mathrm{mg}$ (\#) or $15 \mathrm{mg}$ (\# \# \#) or quinagolide at a daily dose of $225 \mu \mathrm{g}$ (\# \#).

mental treatment center (data not shown). Finally, the median dose of cabergoline was $3.5 \mathrm{mg}$ in patients with psychiatric relapses and $1.375 \mathrm{mg}$ in patients who did not relapse but the difference was not significant $(P=0.1)$.

Other endpoints of psychiatric worsening such as antipsychotic changes were observed in eight patients who were generally the same patients as those who were admitted for psychiatric relapses (Table 1).

Therefore, the causal relationship between the use of DAs and the worsening of psychosis (the patients being treated or not with antipsychotics) remains uncertain.

Details about time course of DA introduction and psychiatric history are given for each patient in Supplemental data.

The fact that a patient had an underlying mental disorder modified the therapeutic decision regarding the adenoma in only one patient (Patient \#11). As his behavior disorders have been presumably induced by bromocriptine, which was prescribed while the patient was not taking any antipsychotic drug, radiotherapy was preferred to reintroduction of dopamine agonists. Bromocriptine was, however, highly effective in decreasing PRL levels and adenoma volume. Of the other four patients who were admitted to the hospital for acute decompensation of their mental disorder under DAs, the dose of DAs was decreased and the type of antipsychotic changed in three patients (Patients \#1, \#3, and \#14). In the last patient (Patient \#8), the acute psychotic episode led to the diagnosis of a masked psychotic disorder that was previously unknown; antipsychotic drug was thus initiated without changing the DA treatment.

None of our patients developed impulse control disorders under dopamine agonists.

\section{Discussion}

In this retrospective study of 18 patients who suffered from both a macroprolactinoma and a severe psychiatric disease justifying treatment with antipsychotics, DAs were generally effective on PRL levels and, to a lesser extent, on tumor volume and visual defects, despite a large disparity in clinical situations and therapeutic strategies.

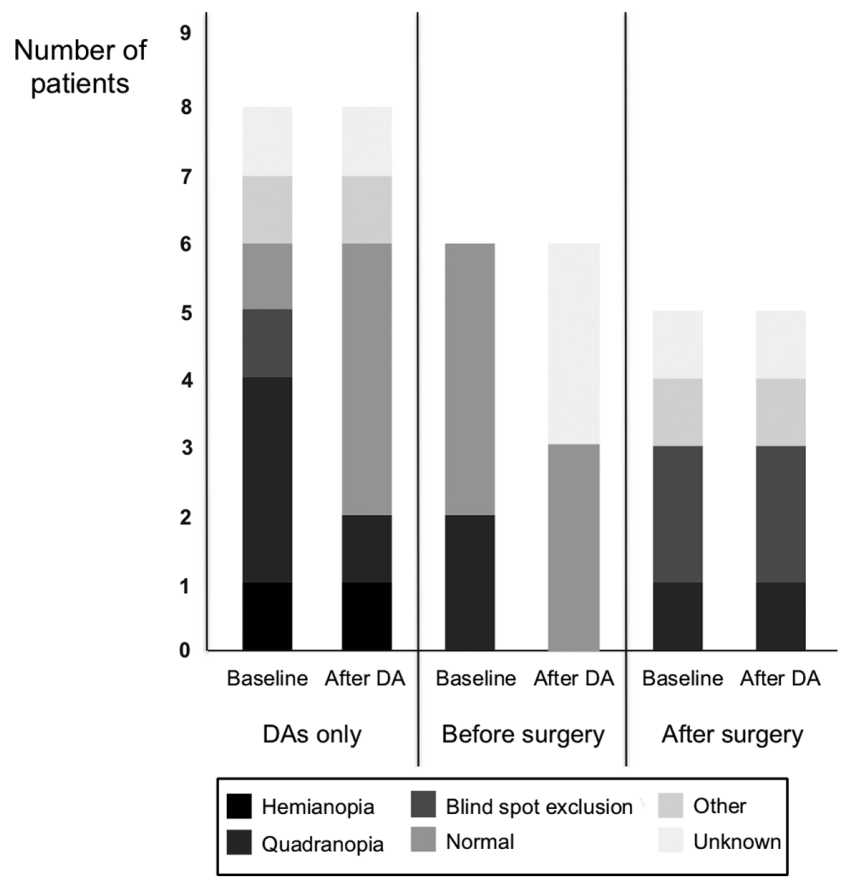

Figure 4

Evolution of the visual field under DA. 
Few psychiatric relapses were observed and none can be definitively imputed to the prescription of DAs.

Two-thirds of our patients were males, which was not unexpected with regard to the male predominance for both psychotic disorders $(2,3)$ and larger macroprolactinomas (1). The age of our patients also corresponds to that reported in epidemiological studies of psychotic disorders $(2,3)$.

In a large retrospective study of 225 patients with a macroprolactinoma from one of our centers, normalization of PRL levels was achieved in $60.8 \%$ of the patients at a median weekly cabergoline dose of 1 mg (14). In the present series of patients with underlying mental disorders necessitating antipsychotics, normal PRL levels were achieved in only $25 \%$ of the patients who were treated with DAs alone and the median dose of cabergoline at the best biochemical control was similar (1 mg per week). This lower efficacy compared to the nonpsychiatric population may have three explanations. A first pathophysiological hypothesis could be a partial resistance of prolactinoma lactotroph cells to DAs related to concomitant D2R blockade by antipsychotics, which act as D2R antagonists (6). A second explanation could be the reluctance of endocrinologists to increase the dose of DAs as they would have done in typical patients with prolactinoma for fear of decompensating the underlying psychotic disorder. This could have led the endocrinologists not to increase the dose and to accept persistently increased PRL levels if the objective of tumor shrinkage was achieved. However, against this hypothesis, one may underline that the median dose of cabergoline used in the three groups of our patients is close to that used in patients without psychotic disorders. Finally, one may question the compliance regarding DAs in these patients with psychotic disorders - indeed, drug compliance to treatment is a major issue in these patients (4).

The most important result of our retrospective study is the efficacy of DAs on macroprolactinoma volume. Indeed, despite the potential resistance to DAs induced by concomitant treatment with antipsychotics, and even if the effect on tumor volume seems less impressive than that observed in 'usual' patients with macroprolactinoma, DAs were able to shrink macroprolactinomas in a high proportion of our patients (57\% in the whole cohort and $87.5 \%$ in those only treated with DAs), quite similarly to the high rate of responders seen in 'usual' patients with macroprolactinoma $(1,15)$. However, the importance of shrinkage might be inferior. Indeed, out of 127 patients treated for more than 1 year by cabergoline alone $(16,17$, 18), 86 (68\%) experienced a greater than 50\% tumor size reduction (vs $17 \%$ in our series), 28 (22\%) showed a $25 \%$ to $50 \%$ reduction (vs $11 \%$ in our series), and $13(10 \%)$ showed a less than 25\% reduction in tumor size (vs 39\% in our series). Such an ability to shrink macroprolactinomas in these patients is particularly interesting in cases of visual defects related to optic pathway compression. Optic chiasm decompression under DA was indeed observed in $44 \%$ of our patients who initially had chiasm compression, and $50 \%$ of the patients who had visual defects normalized their visual fields and acuity. Thus, the main objective of DA treatment, which was to relieve the mass effects of macroprolactinoma, was achieved, even if PRL levels were not normalized in some of them.

This therapeutic issue, that is, the efficacy and tolerance of DA treatment in patients with macroprolactinoma under antipsychotic drug treatment for underlying psychosis, has rarely been addressed. In the literature published since 1985, we found only eight case reports of patients in whom a diagnosis of macroprolactinoma was made while they were taking antipsychotics, and they were all published in psychiatry journals $(10,11$, $12,13,19,20)$. Half of the patients underwent surgery as a first-line treatment. Among the six patients who underwent surgery, four needed adjuvant therapy with DAs. Two patients only received DAs. A similar response to DAs on PRL levels, especially in those only treated with DAs, but a minor efficacy on tumor volume was reported. Unfortunately, endocrinological information is often limited.

Another important issue, in addition to their clinical efficacy, is knowing whether or not adding DAs to antipsychotics may decompensate psychiatric status. Less than $30 \%$ of our patients underwent a noteworthy relapse while taking DAs, and these relapses occurred in the patients who already had a history of psychiatric admission (89\% vs 11\%) and who suffered from the most severe psychiatric diseases. One can thus reasonably question the role of DAs in relapses. Psychiatric relapses led to decrease in the dose of DA in four of our patients. Unfortunately, as the DA dose reduction was concomitant of antipsychotic change, it is difficult to interpret its effect on psychiatric relapse.

To our knowledge, such an acute psychiatric crisis has been reported in 13 patients who had either a micro- or a macroprolactinoma treated with DAs. In four cases, the underlying psychosis was already diagnosed and treated and relapsed at the time of DA treatment $(10,11,12,13)$; in seven cases, psychosis was revealed at this time $(21,22$, $23,24,25,26)$. However, as all the cases were published in psychiatry literature, there might be a publication bias 
overestimating the proportion of psychosis and relapses. Indeed, in a large series of 455 nonpsychiatric patients, no psychosis was diagnosed under cabergoline (27).

Another clinical situation that may help to increase knowledge about the concomitant prescription of antipsychotics and DAs is the population of psychiatric patients with antipsychotic-induced hyperprolactinemia in whom DA was initiated to treat hyperprolactinemia. No psychosis relapse was observed, either in a study involving 80 patients with schizophrenia treated with cabergoline (28) or in several series of patients with psychosis treated with various DAs $(29,30,31,32)$. Reports of psychiatric symptoms worsening in patients with psychosis treated with DAs for antipsychoticinduced hyperprolactinemia are very few $(23,33,34)$ : of a total of 129 patients with psychosis with antipsychoticinduced hyperprolactinemia, only four (3\%) presented exacerbation of their psychiatric symptoms under DA treatment, confirming the good psychiatric tolerance of DAs in this setting.

In clinical situations where continuation of DAs is necessary despite psychiatric relapse induction, a solution could be to treat psychosis with aripiprazole, an antipsychotic with a lower affinity for D2R and a partial agonistic effect on D2R $(10,22,35)$. Other antipsychotics such as clozapine may also be interesting to use in this context as, at a clinically effective dose, it occupies only $40-50 \%$ of D2R and binds more closely to them (fast dissociation).

Finally, none of our patient developed impulse control disorders when given DAs $(36,37)$

Our study has many limitations. First, it is retrospective with all the bias associated with retrospective studies. However, given the scarcity of the association of these two disorders and the time needed to determine the effect and tolerance of DAs in patients with psychosis, a prospective study is impossible to perform, and we need to limit our information to retrospective data. Given the heterogeneity of endocrinological and psychiatric situations, another limitation of our study was the absence of standardization of the data, preventing their objective interpretation. The third limitation is the very large period covered by the study (from the 1980s to 2017), explaining the variety of therapeutic strategies and the high proportion of surgery. DAs have also evolved, and cabergoline, which is currently considered the first choice drug, is better tolerated and allows better compliance to treatment than bromocriptine $(1,38)$. Finally, antipsychotics have also improved and new classes of drugs have been developed, with less hyperprolactinemic effects $(4,5,9)$.

Despite these limitations, we provide the largest study of these rare patients with psychosis (necessitating antipsychotics) and macroprolactinoma (necessitating DAs) in whom interaction of these drugs (both necessary for the patient) was considered pragmatically and from an endocrine point of view. The multicenter aspect of our study provides a representative idea of how these patients are treated in France and Belgium and allows some extrapolation of the results.

\section{Conclusion}

In conclusion, according to the results of our retrospective study, the benefit-risk ratio of initiating DAs seems favorable in patients suffering from a macroprolactinoma with mass effects while they are treated with antipsychotics for a stable psychiatric disease. In absence of visual involvement, severe headaches or invasion into the skull base, a wait-and-see strategy may be proposed, provided sexual steroids are given. Otherwise, a treatment is necessary. Cabergoline, which is currently the first-line DA, proved to be effective even if its combination with antipsychotics seems to induce a partial resistance to DA related to D2R blockade by antipsychotics. At the dose used (sometimes insufficient to achieve normal PRL levels), the effect on macroprolactinoma volume is comparable to what is observed in nonpsychiatric patients. This is very helpful in these difficult patients who often refuse surgery. Very importantly, DA treatment in this context does not seem to induce relapse of underlying stable psychiatric disorders. Thus, surgery should be avoided when a good response to DAs is achieved on PRL levels and tumor volume. If psychosis is more unstable, multidisciplinary discussion between the endocrinologist, the psychiatrist and the neurosurgeon is indicated for deciding the therapeutic strategy.

\section{Supplementary materials}

This is linked to the online version of the paper at https://doi.org/10.1530/ EJE-20-0125.

\section{Declaration of interest}

The authors declare that there is no conflict of interest that could be perceived as prejudicing the impartiality of this study. 


\section{Funding}

This research did not receive any specific grant from any funding agency in the public, commercial or not-for-profit sector.

\section{Acknowledgements}

The authors wish to thank Tristan Verdelet for his help in drawing the figures.

\section{References}

1 Chanson P \& Maiter D. The epidemiology, diagnosis and treatment of prolactinomas: the old and the new. Best Practice and Research: Clinical Endocrinology and Metabolism 201933 101290. (https://doi. org/10.1016/j.beem.2019.101290)

2 Jongsma HE, Turner C, Kirkbride JB \& Jones PB. International incidence of psychotic disorders, 2002-17: a systematic review and meta-analysis. Lancet Public Health 20194 e229-e244. (https://doi. org/10.1016/S2468-2667(19)30056-8)

3 Moreno-Kustner B, Martin C \& Pastor L. Prevalence of psychotic disorders and its association with methodological issues. A systematic review and meta-analyses. PLoS One 201813 e0195687. (https://doi. org/10.1371/journal.pone.0195687)

4 Lieberman JA \& First MB. Psychotic disorders. New England Journal of Medicine 2018379 270-280. (https://doi.org/10.1056/ NEJMra1801490)

5 Miyamoto S, Miyake N, Jarskog LF, Fleischhacker WW \& Lieberman JA. Pharmacological treatment of schizophrenia: a critical review of the pharmacology and clinical effects of current and future therapeutic agents. Molecular Psychiatry 201217 1206-1227. (https:// doi.org/10.1038/mp.2012.47)

6 Molitch ME. Drugs and prolactin. Pituitary 200811 209-218. (https://doi.org/10.1007/s11102-008-0106-6)

7 Turrone P, Kapur S, Seeman MV \& Flint AJ. Elevation of prolactin levels by atypical antipsychotics. American Journal of Psychiatry 2002 159 133-135. (https://doi.org/10.1176/appi.ajp.159.1.133)

8 Holt RI \& Peveler RC. Antipsychotics and hyperprolactinaemia: mechanisms, consequences and management. Clinical Endocrinology 201174 141-147. (https://doi.org/10.1111/j.13652265.2010.03814.x)

9 Lieberman JA, Stroup TS, McEvoy JP, Swartz MS, Rosenheck RA, Perkins DO, Keefe RS, Davis SM, Davis CE, Lebowitz BD et al. Effectiveness of antipsychotic drugs in patients with chronic schizophrenia. New England Journal of Medicine 2005353 1209-1223. (https://doi.org/10.1056/NEJMoa051688)

10 Melkersson K \& Hulting AL. Prolactin-secreting pituitary adenoma in neuroleptic treated patients with psychotic disorder. European Archives of Psychiatry and Clinical Neuroscience 2000250 6-10. (https://doi.org/10.1007/pl00007539)

11 Broekhof R, Gosselink MJ, Pijl H \& Giltay EJ. The effect of aripiprazole and quinagolide, a dopamine agonist, in a patient with symptomatic pituitary prolactinoma and chronic psychosis. General Hospital Psychiatry 201234 209.e1-209.e3. (https://doi.org/10.1016/j. genhosppsych.2011.07.004)

12 Pal JK \& Sarino WA. Effect of risperidone on prolactinoma growth in a psychotic woman. Psychosomatic Medicine 200062 736-738. (https://doi.org/10.1097/00006842-200009000-00018)

13 Weingarten JC \& Thompson TL, 2nd. The effect of thioridazine on prolactinoma growth in a schizophrenic man: case report. General Hospital Psychiatry 19857 364-366. (https://doi.org/10.1016/01638343(85)90053-2)

14 Paepegaey AC, Salenave S, Kamenicky P, Maione L, Brailly-Tabard S, Young J \& Chanson P. Cabergoline tapering is almost always successful in patients with macroprolactinomas. Journal of the
Endocrine Society 20171 221-230. (https://doi.org/10.1210/js.201700038)

15 Gillam MP, Molitch ME, Lombardi G \& Colao A. Advances in the treatment of prolactinomas. Endocrine Reviews 200627 485-534. (https://doi.org/10.1210/er.2005-9998)

16 Chanson P \& Maiter D. Prolactinoma. In The Pituitary, 4th ed, pp 467-514. Ed S Melmed. London, UK: Elsevier, 2017.

17 Colao A, Di Sarno A, Landi ML, Scavuzzo F, Cappabianca P, Pivonello R, Volpe R, Di Salle F, Cirillo S, Annunziato L et al. Macroprolactinoma shrinkage during cabergoline treatment is greater in naive patients than in patients pretreated with other dopamine agonists: a prospective study in 110 patients. Journal of Clinical Endocrinology and Metabolism 200085 2247-2252. (https:// doi.org/10.1210/jcem.85.6.6657)

18 Delgrange E, Daems T, Verhelst J, Abs R \& Maiter D. Characterization of resistance to the prolactin-lowering effects of cabergoline in macroprolactinomas: a study in 122 patients. European Journal of Endocrinology 2009160 747-752. (https://doi.org/10.1530/EJE-090012)

19 Ali S, Miller KK \& Freudenreich O. Management of psychosis associated with a prolactinoma: case report and review of the literature. Psychosomatics 201051 370-376. (https://doi.org/10.1176/ appi.psy.51.5.370)

20 Santos Andrade EH, Pan PM, da Silva PF \& Gadelha A. New insights in the management of antipsychotics in the treatment of schizophrenia in a patient with prolactinoma: a case report and review of the literature. Case Reports in Medicine 20102010573252. (https://doi.org/10.1155/2010/573252)

21 Bilal L \& Ching C. Cabergoline-induced psychosis in a patient with undiagnosed depression. Journal of Neuropsychiatry and Clinical Neurosciences 201224 E54. (https://doi.org/10.1176/appi. neuropsych.11110348)

22 Burback L. Management of a microprolactinoma with aripiprazole in a woman with cabergoline-induced mania. Endocrinology, Diabetes and Metabolism Case Reports 20152015 150100. (https://doi. org/10.1530/EDM-15-0100)

23 Frye PE, Pariser SF, Kim MH \& O'Shaughnessy RW. Bromocriptine associated with symptom exacerbation during neuroleptic treatment of schizoaffective schizophrenia. Journal of Clinical Psychiatry 198243 252-253.

24 Mohapatra S \& Nayak MR. Cabergoline-induced mania in a patient of pituitary microadenoma. Indian Journal of Psychological Medicine 201739 350-351. (https://doi.org/10.4103/0253-7176.207314)

25 Nieman DH, Sutterland AL, Otten J, Becker HE, Drent ML, van der Gaag M, Birchwood M \& de Haan L. Treating prolactinoma and psychosis: medication and cognitive behavioural therapy. BMJ Case Reports 2011 2011. (https://doi.org/10.1136/bcr.07.2010.3185)

26 Peter SA, Autz A \& Jean-Simon ML. Bromocriptine-induced schizophrenia. Journal of the National Medical Association $1993 \mathbf{8 5}$ 700-701.

27 Verhelst J, Abs R, Maiter D, van den Bruel A, Vandeweghe M, Velkeniers B, Mockel J, Lamberigts G, Petrossians P, Coremans P et al. Cabergoline in the treatment of hyperprolactinemia: a study in 455 patients. Journal of Clinical Endocrinology and Metabolism 199984 2518-2522. (https://doi.org/10.1210/jcem.84.7.5810)

28 Kalkavoura CS, Michopoulos I, Arvanitakis P, Theodoropoulou P, Dimopoulou K, Tzebelikos E \& Lykouras L. Effects of cabergoline on hyperprolactinemia, psychopathology, and sexual functioning in schizophrenic patients. Experimental and Clinical Psychopharmacology 201321 332-341. (https://doi.org/10.1037/a0033448)

29 Cavallaro R, Cocchi F, Angelone SM, Lattuada E \& Smeraldi E. Cabergoline treatment of risperidone-induced hyperprolactinemia: a pilot study. Journal of Clinical Psychiatry 200465 187-190. (https:// doi.org/10.4088/jcp.v65n0207)

30 Cohn JB, Brust J, DiSerio F \& Singer J. Effect of bromocriptine mesylate on induced hyperprolactinemia in stabilized psychiatric 
outpatients undergoing neuroleptic treatment. Neuropsychobiology 198513 173-179. (https://doi.org/10.1159/000118184)

31 Pollock A \& McLaren EH. Serum prolactin concentration in patients taking neuroleptic drugs. Clinical Endocrinology 199849 513-516. (https://doi.org/10.1046/j.1365-2265.1998.00569.x)

32 Tollin SR. Use of the dopamine agonists bromocriptine and cabergoline in the management of risperidone-induced hyperprolactinemia in patients with psychotic disorders. Journal of Endocrinological Investigation 200023 765-770. (https://doi. org/10.1007/BF03345068)

33 Chang SC, Chen CH \& Lu ML. Cabergoline-induced psychotic exacerbation in schizophrenic patients. General Hospital Psychiatry 200830 378-380. (https://doi.org/10.1016/j. genhosppsych.2007.11.002)

34 Smith S. Neuroleptic-associated hyperprolactinemia. Can it be treated with bromocriptine? Journal of Reproductive Medicine 199237 737-740.

35 Hoffer ZS, Roth RL \& Mathews M. Evidence for the partial dopaminereceptor agonist aripiprazole as a first-line treatment of psychosis in patients with iatrogenic or tumorogenic hyperprolactinemia. Psychosomatics 200950 317-324. (https://doi.org/10.1176/appi. psy.50.4.317)

36 Ioachimescu AG, Fleseriu M, Hoffman AR, Vaughan III TB \& Katznelson L. Psychological effects of dopamine agonist treatment in patients with hyperprolactinemia and prolactin-secreting adenomas. European Journal of Endocrinology 2019180 31-40. (https://doi. org/10.1530/EJE-18-0682)

37 Dogansen SC, Cikrikcili U, Oruk G, Kutbay NO, Tanrikulu S, Hekimsoy Z, Hadzalic A, Gorar S, Omma T, Mert M et al. Dopamine agonist-induced impulse control disorders in patients with prolactinoma: a cross-sectional multicenter study. Journal of Clinical Endocrinology and Metabolism 2019104 2527-2534. (https://doi. org/10.1210/jc.2018-02202)

38 Webster J. A comparative review of the tolerability profiles of dopamine agonists in the treatment of hyperprolactinaemia and inhibition of lactation. Drug Safety 199614 228-238. (https://doi. org/10.2165/00002018-199614040-00003)

Received 18 February 2020

Revised version received 4 May 2020

Accepted 1 June 2020 